\title{
Size-Effect Testing of Cohesive Fracture Parameters and Nonuniqueness of Work-of-Fracture Method
}

\author{
Zdeněk P. Bažant, Hon.M.ASCE${ }^{1}$; and Qiang Yu²
}

\begin{abstract}
The cohesive crack model has been widely accepted as the best compromise for the analysis of fracture of concrete and other quasibrittle materials. The softening stress-separation law of this model is now believed to be best described as a bilinear curve characterized by four parameters: the initial and total fracture energies $G_{f}$ and $G_{F}$, the tensile strength $f_{t}^{\prime}$, and the knee-point ordinate $\sigma_{1}$. The classical work-of-fracture test of a notched beam of one size can deliver a clear result only for $G_{F}$. Here it is shown computationally that the same complete load-deflection curve can be closely approximated with stress-separation curves in which the $f_{t}^{\prime}$ values differ by $77 \%$ and $G_{f}$ values by $68 \%$. It follows that the work-of-fracture test alone cannot provide an unambiguous basis for quasibrittle fracture analysis. It is found, however, that if this test is supplemented by size-effect testing, all four cohesive crack model parameters can be precisely identified and the fracture analysis of structures becomes unambiguous. It is shown computationally that size-effect tests do not suffice for determining $G_{F}$ and $f_{t}^{\prime}$, which indicates that they provide a sufficient basis for computing neither the postpeak softening of fracturing structures nor the peak loads of a very large structure. However, if the size-effect tests are supplemented by one complete softening loaddeflection curve of a notched specimen, an unambiguous calculation of peak loads and postpeak response of structures becomes possible. To this end, the notched specimen tests must be conducted in a certain size range, whose optimum is here established by extending a previous analysis. Combination of the work-of-fracture and size-effect testing could be avoided only if the ratios $G_{F} / G_{f}$ and $\sigma_{1} / f_{t}^{\prime}$ were known a priori, but unfortunately their estimates are far too uncertain. DOI: 10.1061/(ASCE)EM.1943-7889.0000254. @ 2011 American Society of Civil Engineers.
\end{abstract}

CE Database subject headings: Cracking; Size effect; Parameters.

Author keywords: Quasibrittle fracture; Cohesive crack model; Fracture mechanics; Cracks; Quasibrittle materials; Concrete; Scaling; Testing; Brittleness.

\section{Introduction}

Fracture behavior of concrete or other quasibrittle materials is manifested in two basic phenomena: (1) a gradual postpeak softening in the load-deflection curve, and (2) a size effect on the nominal strength of geometrically similar structures, representing a gradual transition from no size effect at very small sizes to the strong size effect of linear elastic fracture mechanics (LEFM) at very large sizes. Measurement of these two phenomena is the way to identify the material characteristics of quasibrittle fracture.

During the last decade, it has been widely accepted that a realistic characterization of material fracture properties is provided by the cohesive crack model, first proposed (for a different purpose) by Barenblatt $(1959,1962)$, rigorously formulated by Rice (1968), and pioneered for concrete by Hillerborg et al. (1976) under the alternative name "fictitious crack model." For several decades, it was thought that the cohesive crack model can be adequately calibrated by the work-of-fracture test.

\footnotetext{
${ }^{1}$ McCormick Institute Professor and W. P. Murphy Professor of Civil Engineering and Materials Science, Northwestern Univ., 2145 Sheridan Rd., CEE/A135, Evanston, IL 60208 (corresponding author). E-mail: z-bazant@northwestern.edu

${ }^{2}$ Postdoctoral Research Associate, Northwestern Univ., 2145 Sheridan Rd., CEE/A135, Evanston, IL 60208.

Note. This manuscript was submitted on September 15, 2010; approved on February 10, 2011; published online on July 15, 2011. Discussion period open until January 1, 2012; separate discussions must be submitted for individual papers. This paper is part of the Journal of Engineering Mechanics, Vol. 137, No. 8, August 1, 2011. CASCE, ISSN 0733-9399/2011/ $8-580-588 / \$ 25.00$.
}

In this test, proposed for ceramics by Nakayama (1965) and Tatersall and Taplin (1966), pioneered for concrete by Hillerborg et al. (1976) and Petersson (1981), and standardized by RILEM (1985), notched concrete beams of one size are tested to obtain the complete load-deflection curve, including the postpeak softening (see in detail Bažant and Planas 1998). Hints that this test is not perfect came from observations that the fracture energy $G_{F}$ apparently depends on the specimen size, which would, of course, defeat the concept of fracture energy as a material parameter. This study will show that, to obtain unique results, the work-of-fracture test must be combined with the testing of size effect.

Until the 1980s, it was generally assumed that when a size effect is observed, it must be the Weibull $(1939,1951)$ statistical size effect caused by spatial material randomness. However, when a specimen is notched, such randomness cannot affect the mean strength, and when the structure reaches the maximum load only after stable growth of a large crack (as is typical of reinforced concrete), the mean size effect attributed to material randomness must be negligible because the crack tip location is dictated by mechanics, making it impossible for the crack tip to sample material volumes with random differences in strength. A nonstatistical size effect in notched specimens of concrete was experimentally demonstrated by Walsh $(1972,1976)$. Its necessity was theoretically shown by Bažant (1976, 1977a, b, 1978), and extensive computational demonstration with a material damage model was given by Bažant and Oh (1983).

A simple, theoretically derived, approximate formula for the nonstatistical size effect followed (Bažant 1984, 1997, 2004, 2005). Adopted for a standardized test by RILEM (1990), and later extended to include the geometry (or shape) effects by 
Bažant and Kazemi (1990a), this formula, called the size-shape effect law (SEL) or simply size-effect law, reads

$$
\sigma_{N}=\frac{B f_{t}^{\prime}}{\sqrt{1+D / D_{0}}}=\sqrt{\frac{E G_{f}}{g_{0}^{\prime} c_{f}+g_{0} D}}
$$

where $D=$ characteristic structure size (chosen here as the beam depth); $B=$ dimensionless constant depending on structure geometry; $f_{t}^{\prime}=$ material tensile strength; $E=$ Young's elastic modulus; $g_{0}=g\left(\alpha_{0}\right)=k^{2}\left(\alpha_{0}\right)=$ dimensionless energy release function of LEFM evaluated at notch tip $\alpha_{0}$, which introduces the effect of structure geometry (or shape); $k\left(\alpha_{0}\right)=$ dimensionless stress intensity factor; $g_{0}^{\prime}=$ first derivative of function $g$ evaluated at notch tip; $\alpha_{0}=a_{0} / D ; a_{0}=$ depth of notch (or initial crack); $G_{f}=$ initial fracture energy; and $c_{f}=$ material length constant representing the distance from the tip of notch (or crack) to the resultant of tensile cohesive stresses $\sigma$ in a fully developed fracture process zone (FPZ). Finally, for two-dimensional similarity, $\sigma_{N}=P / b D=$ nominal strength of structure or specimen $=$ parameter of maximum load $P$ having the dimension of stress; and $b=$ structure width [note that Eq. (1) applies only to structures with large enough notches or cracks, which is called the Type 2 size effect; another formula applies to Type 1 size effect, which occurs for failures at crack initiation from a smooth surface (Bažant 2005; Yu et al. 2010)].

According to Eq. (1), for $D \ll D_{0}$ the failure is quasi-plastic, and the size effect on $\sigma_{N}$ disappears, and for $D \gg D_{0}$, the failure is brittle, and the size effect of LEFM, which is the strongest possible, is approached. Exploiting the plastic-brittle transition of size effect described by Eq. (1) makes it possible to determine two fracture characteristics from the size-effect tests, $G_{f}$ and $c_{f}$ (Bažant and Kazemi 1990a, b, 1991; Bažant and Planas 1998; Bažant 2005; Bažant et al. 2002; RILEM TC QFS 2004).

Unfortunately, the fracture parameters obtained from the sizeeffect tests (i.e., maximum load tests of specimens of sufficiently different sizes) are also incomplete-similar to those obtained from the work-of-fracture test, but in a different way. Because of the prohibitive cost of testing specimens sufficiently larger than the length of the FPZ in concrete (which is approximately $0.6 \mathrm{~m}$, or $2 \mathrm{ft}$, for normal concretes), the size-effect tests are insufficient to predict the maximum loads of very large structures. Furthermore, it has been long known that the size-effect tests are also insufficient for accurate prediction of the postpeak softening of the load-deflection curve, which is particularly important for the analysis of dynamic failures.

This study will show that, to overcome the aforementioned limitations, the size-effect testing must be supplemented by the work-of-fracture test.

\section{Size-Effect Testing of Notched Concrete Beams}

Experimental identification of the softening stress-separation function $\sigma(w)$ of the cohesive (or fictitious) crack model is a complex problem. A direct measurement of this function is impossible. The only way is an inverse analysis of fracture tests of notched specimens.

It is now generally accepted that the stress-separation law $\sigma(w)$ is not linear. A realistic approximation is a bilinear curve with a long tail. It is characterized by four independent key parameters: The initial and total fracture energies, $G_{f}$ and $G_{F}$, representing the areas under the initial linear segment and under the entire curve; the tensile strength $f_{t}^{\prime}$; and the stress $\sigma_{1}$ at the knee point (see Fig. 1). The problem is how to identify these key parameters from tests.

For the size range normally used in concrete testing, a fully de-

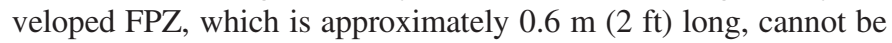

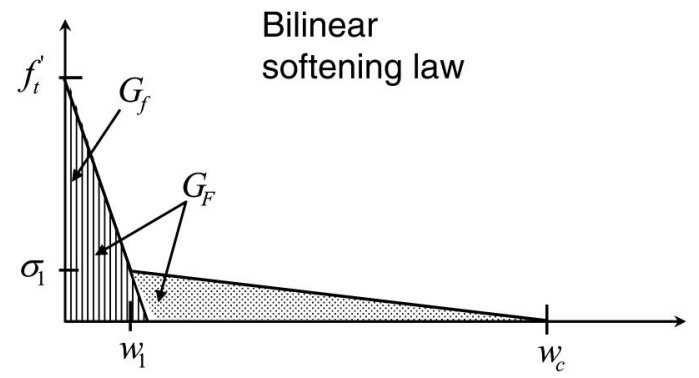

Fig. 1. Bilinear softening law of cohesive crack model

achieved at peak load. As a consequence, the cohesive stress in the FPZ at peak load still lies completely within the initial steeply descending linear portion of the bilinear $\sigma(w)$ curve. So, the tail of the softening $\sigma(w)$ cannot play any significant role in the maximum load. Therefore no information about the tail, which is important to determine the total fracture energy $G_{F}$, can be obtained by analyzing the peak load alone, unless impracticably large concrete specimens (i.e., $D \geq 5 \mathrm{~m}$, or $16 \mathrm{ft}$ ) were tested.

This indicates that, to obtain information on the total fracture energy $G_{F}$, the testing of the size effect on the maximum loads must be supplemented by postpeak deflection measurements. Unfortunately, size-effect test data that include the postpeak deflections have been far too limited. To fill this gap, tests of this kind have recently been carried out at Northwestern University by using three-point bend beams geometrically similar in two dimensions, of beam depths $D=40 \mathrm{~mm}$ (1.57 in.), $93 \mathrm{~mm}$ (3.66 in.), and $215 \mathrm{~mm}$ (8.46 in.), with notches of depth $0.15 \mathrm{D}$ and span-to-depth ratio 2.4. The concrete had mean Young's modulus $E=30 \mathrm{GPa}$ $(4,350 \mathrm{ksi})$ and mean compressive strength $f_{c}^{\prime}=41 \mathrm{MPa}$ $(5,945 \mathrm{psi})$. The complete postpeak load-crack mouth opening displacement (CMOD) curves were recorded for the largest specimens, and partial ones for the medium-size specimens. The test details are reported in Yu et al. (2010) (except for the fact that the MTS machine used for the largest specimen was much stiffer than the one used for the medium and small specimens).

A finite-element code based on the cohesive crack model was used to fit, for all the sizes, the measured maximum loads and postpeak deflections ( $\mathrm{Yu}$ et al. 2010). The following bilinear $\sigma(w)$ parameters, defined in Fig. 1, were identified: $f_{t}^{\prime}=4.80 \mathrm{MPa}$ (696 psi), $w_{c}=0.08 \mathrm{~mm}\left(0.00315\right.$ in.), $\sigma_{1}=0.2 f_{t}^{\prime}=0.96 \mathrm{MPa}$ (139.2 psi), and $w_{1}=0.0107 \mathrm{~mm}(0.000421 \mathrm{in}$.$) . For the afore-$ mentioned parameters, the coefficient of variation (COV) of errors of the numerical simulations compared to the mean load-CMOD curve of the four specimens is quite small; $\mathrm{CoV}=3.9 \%$. For the errors compared with the mean peak loads of specimens of three different sizes, $\mathrm{CoV}=2.2 \%$. Figs. 2(a) and 2(b) document that excellent fits were obtained; see also Yu et al. (2010).

If $f_{t}^{\prime}$ and $G_{f}$ (or $G_{F}$ ) are known, the bilinear softening function $\sigma(w)$ is fixed by the ratio $G_{F} / G_{f}$ and the ordinate $\sigma_{1}$ of the knee point. If the tail is deleted [i.e., if $\sigma(w)$ becomes a linear cohesive law], or if its slope is reduced (i.e., if $G_{F}$ is increased), then the peak load values $\sigma_{N}$ for the three sizes can still be matched quite closely, but the load-CMOD curve will deviate substantially after the peak load [see Fig. 2(a)]. The explanation is that the cohesive stresses in the FPZ do not enter the tail within the normal size range of tests (see Fig. 3, which shows the cohesive stress at notch tip at peak load for different sizes).

Although the tail plays a significant role in the postpeak curve, it has a negligible effect on the peak load. If the stress profile along the ligament at peak load is plotted for specimens of $D=40 \mathrm{~mm}$ and $215 \mathrm{~mm}$ (1.57 in. and $8.46 \mathrm{in}$.), it can be seen from Fig. 3 that 
(a)

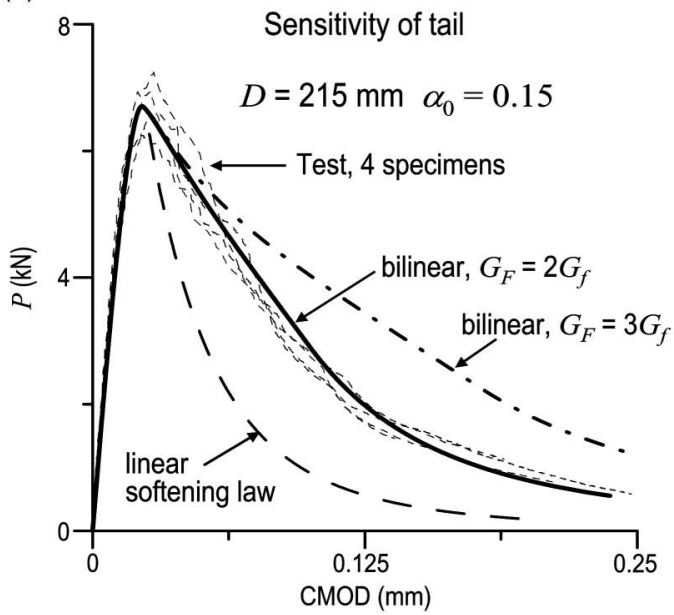

(b)

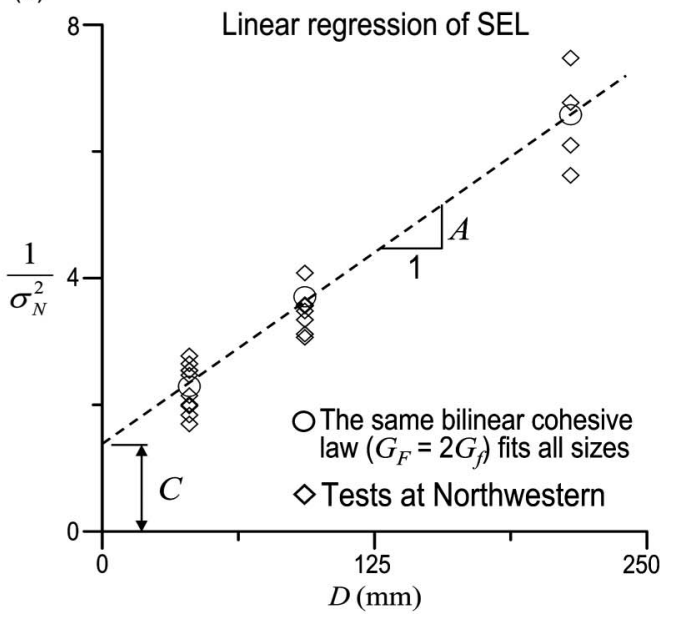

Fig. 2. (a) Simulations compared with load-CMOD curves measured for $D=215 \mathrm{~mm}$ (8.46 in.); and (b) peak loads obtained by simulations compared with tests

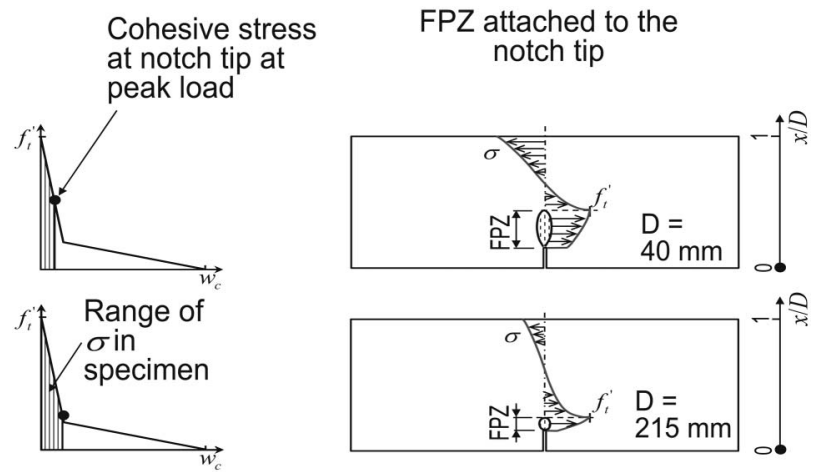

Fig. 3. Cohesive stress and fracture process zone at peak load for sizes used in test

the FPZ, which is located far away from the boundary, is not fully developed and the cohesive stress at the notch tip is higher than $\sigma_{1}$.

\section{Identification of Initial Fracture Energy and Cohesive Tensile Strength from Size Effect}

According to the SEL, the size-effect parameters $G_{f}$ and $c_{f}$ can be determined by rearranging Eq. (1) in the linear regression form $Y=A X+C$, in which $X=D, Y=\sigma_{N}^{-2}, A=g_{0} / E G_{f}$, and $C=g_{0}^{\prime} c_{f} / E G_{f}$ (Bažant and Kazemi 1991; Bažant and Planas 1998). However, to reveal the relation of the size-effect law to the cohesive crack model, it is convenient to rearrange Eq. (1) into a different linear plot, having the slope of 1 (Cusatis and Schauffert 2009; Yu et al. 2010):

$$
\frac{f_{t}^{\prime 2}}{g_{0}^{\prime} \sigma_{N}^{2}}=\frac{c_{f}}{l_{1}}+\frac{g_{0} D}{g_{0}^{\prime} l_{1}} \quad \text { or } \quad Y=\gamma+\bar{D}
$$

where $\gamma=c_{f} / l_{1} ; Y=f_{t}^{\prime 2} / g_{0}^{\prime} \sigma_{N}^{2}=$ dimensionless shape-independent strength parameter; $l_{1}=E G_{f} / f_{t}^{\prime 2}=$ Irwin's (1958) material characteristic length corresponding to $G_{f}$; and $\bar{D}=$ dimensionless (shapeindependent) structure size, defined as

$$
\bar{D}=\frac{D}{l_{1}} \frac{g_{0}}{g_{0}^{\prime}}
$$

Parameter $\gamma$ is a constant for a given cohesive softening law (Cusatis and Schauffert 2009; Yu et al. 2010). This constant varies little with changes in the cohesive softening law, a fact which helps in determining $f_{t}^{\prime}$, the tensile strength of the cohesive crack model, from the size-effect measurements (see Yu et al. 2010). This is important because, unfortunately, the tensile strength value obtained by the direct tensile tests, flexural tests or Brazilian split-cylinder tests can be expected to be only a crude estimate of $f_{t}^{\prime}$ for the cohesive crack model. The reason is that these tests, especially the Brazilian, suffer from various kinds of size effects, and that they produce a rather different strain field in the failure zone.

The relation of size effect to tensile strength has been illuminated by cohesive crack simulations of Bažant et al. (2002), and especially those of Cusatis and Schauffert (2009). The latter investigators showed that, in the case of linear softening (i.e., for $\left.G_{F} / G_{f}=1\right), \gamma=c_{f} / l_{1}=0.44$ for the large-size asymptote of the size-effect plot (Fig. 4). In this and subsequent figures, all the data points are obtained by simulations based on the aforementioned parameters. In the case of bilinear softening, one may distinguish two asymptotes - the final large-size asymptote, which corresponds to $G_{F}$, and the intermediate asymptote in the sense of Barenblatt (1996, 2003), which corresponds to $G_{f}$, i.e., to the initial linear portion of the stress-separation curve (and is approached asymptotically if $\left.l_{c h} / l_{1} \rightarrow \infty\right)$.

For $D / l_{1} \rightarrow \infty$, the cohesive crack simulations asymptotically approach the size-effect regression line of SEL (Bažant et al. 2002), but deviate significantly for small sizes, implying a smaller value of $c_{f}$. To identify the material parameters despite these deviations, a correction is thus necessary.

The regression line of size-effect tests will approach closely enough the final asymptote corresponding to $G_{F}$ only for specimen sizes far beyond the feasible laboratory range (Fig. 4), precisely for $\bar{D}>10$. If the tail is deleted and only the initial softening slope is considered, the asymptote will become much steeper and will be approached closely for sizes an order of magnitude smaller (Fig. 4). These features transpire from cohesive crack model simulations for different specimen sizes and different geometries (particularly, for different $\alpha_{0}$ ), based on the same bilinear softening function $\sigma(w)$ verified by tests; see Fig. 4.

As pointed out in Cusatis and Schauffert (2009) and further supported in Yu et al. (2010), it is by virtue of the transition from the intermediate to the final asymptote that the size-effect curve, as 

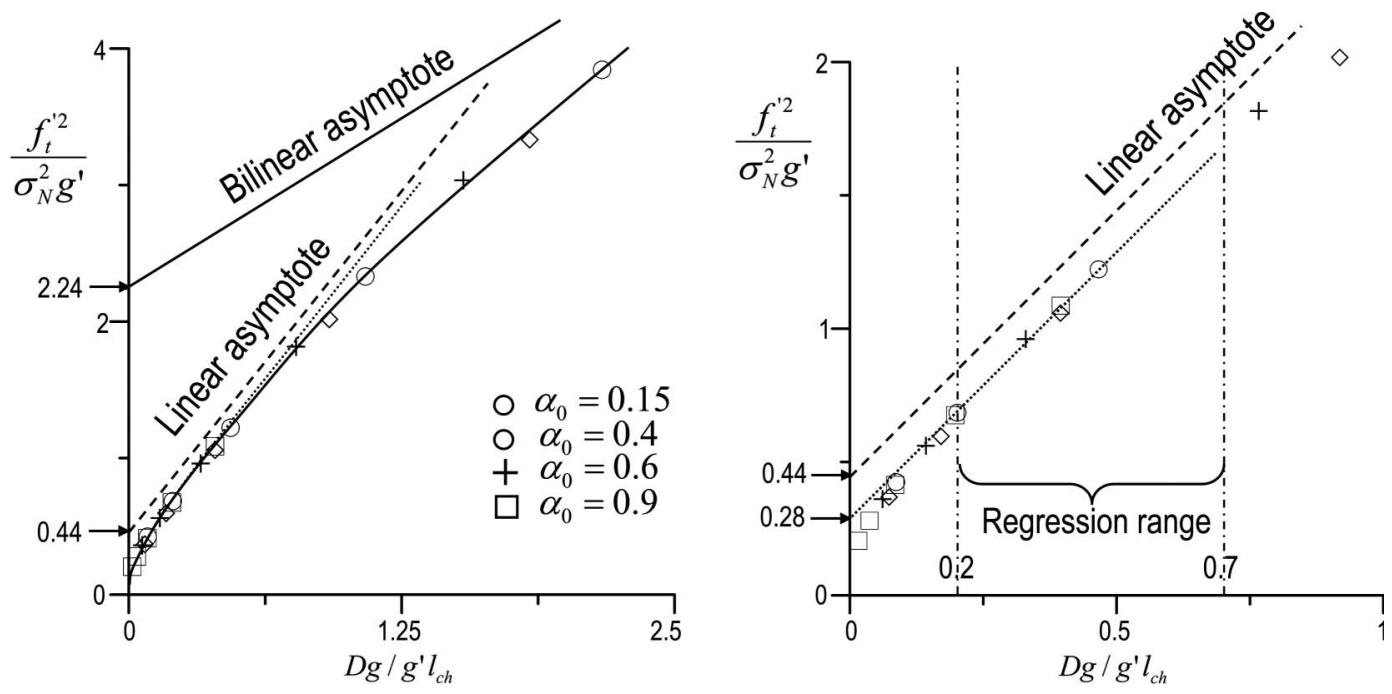

Fig. 4. Simulations based on cohesive crack model and the asymptotes determined by linear and bilinear softening

computed from the cohesive crack model, possesses a segment that is virtually parallel to the asymptote for the linear softening, the slope of which determines the $G_{f}$ value (Fig. 4). This parallel segment luckily occurs within the range of laboratory specimen sizes, which is the reason why the classical size-effect method has always been giving realistic values of $G_{f}$, even though the relationship of SEL to the cohesive crack model was not known.

The downward shift of the regression line of data from the intermediate asymptote (Fig. 4) gives the value of $\gamma$, which must be used to get the correct values of $l_{1}$ and $f_{t}^{\prime}$. Cusatis and Schauffert (2009) found that, for bilinear softening, the asymptotic slope corresponding to $G_{f}$ was best obtained by tests within the range $0.2<\hat{D}=D g / g^{\prime} l_{c h}<0.7$ in which the size-effect plot is visually undistinguishable from a straight line; where $l_{c h}=E G_{F} / f_{t}^{\prime 2}$. It is shown subsequently that $\hat{D}$ should better be replaced with $\bar{D}$ and thus, the optimal size range is

$$
\bar{D} \in(0.4,1.4)
$$

Cusatis and Schauffert (2009) obtained their optimal size range by setting $\sigma_{1} / f_{t}^{\prime}=0.25$ and $G_{F} / G_{f}=2$. They did not examine the possible effects of the ratios $\sigma_{1} / f_{t}^{\prime}$ and $G_{F} / G_{f}$ on the optimal size range for determining $G_{f}$ and $f_{t}^{\prime}$. Changing the ratio $\sigma_{1} / f_{t}^{\prime}$ up and down while keeping $G_{F} / G_{f}$ constant, it is found here that the optimal size range $(0.4<\bar{D}<1.4)$ will not change significantly for the typical test geometry (three-point notched beams of span-to-depth ratios from 2.4 to 8 and notch-depth ratio $\alpha_{0}$ from 0.15 to 0.6 ) when $\sigma_{1} / f_{t}^{\prime} \leq 0.25$. As shown in Figs. 5 and 6 , the slopes of the regression line of size-effect data computed by cohesive crack model for $\sigma_{1} / f_{t}^{\prime}$ ranging from 0.2 to 0.25 are virtually parallel to the theoretical asymptotic slope for linear softening, and the $G_{f}$ value calculated from the linear regression slope [Eq. (2)] agrees with the value in the cohesive crack model very well. For the optimal size range, the maximum error is less than $5 \%$ (see Table 1).

For the average value $\gamma=0.285$ used in Yu et al. (2010), the maximum error of tensile strength $f_{t}^{\prime}$ obtained by linear regression of the cohesive crack model data is only $6 \%$, which is well within the inevitable scatter of testing. However, when $\sigma_{1} / f_{t}^{\prime}=0.3$, the aforementioned size range will not be optimal and would not yield a close estimate of $G_{f}$ (note that in Fig. 7, the regression slope deviates from the asymptotic slope substantially). The error in $G_{f}$ obtained is more than $14 \%$ and in $f_{t}^{\prime}$ more then $11 \%$ (Table 1), which is greater than the scatter in normal testing. The main reason

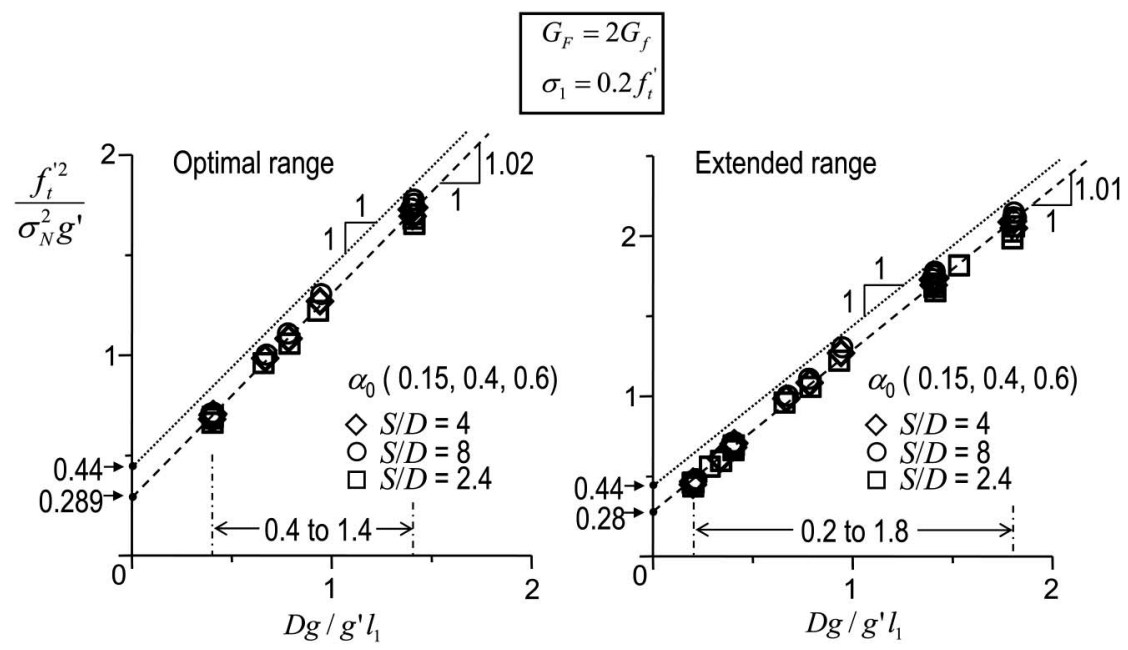

Fig. 5. Slope and intercept obtained by regression for $\sigma_{1}=0.2 f_{t}^{\prime}$ for (a) optimal; and (b) extended size range 


$$
\begin{aligned}
& G_{F}=2 G_{f} \\
& \sigma_{1}=0.25 f_{t}^{\prime}
\end{aligned}
$$
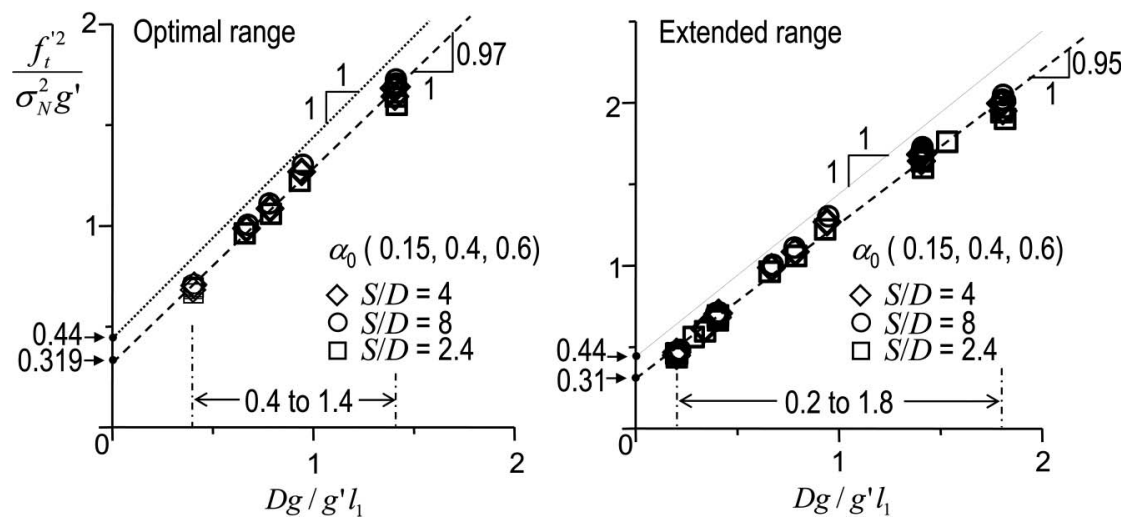

Fig. 6. Slope and intercept obtained by regression for $\sigma_{1}=0.25 f_{t}^{\prime}$ for (a) optimal; and (b) extended size range

Table 1. Effect of $\sigma_{1}$ on Optimal Size Range $\bar{D}, G_{F} / G_{f}=2$

\begin{tabular}{|c|c|c|c|c|c|c|c|c|c|c|c|c|}
\hline \multirow[b]{3}{*}{$\bar{D}$} & \multicolumn{4}{|c|}{$\sigma_{1}=0.2 f_{t}^{\prime}$} & \multicolumn{4}{|c|}{$\sigma_{1}=0.25 f_{t}^{\prime}$} & \multicolumn{4}{|c|}{$\sigma_{1}=0.3 f_{t}^{\prime}$} \\
\hline & \multicolumn{2}{|c|}{$\alpha_{0}=0.15$} & \multicolumn{2}{|c|}{$\alpha_{0}=0.6$} & \multicolumn{2}{|c|}{$\alpha_{0}=0.15$} & \multicolumn{2}{|c|}{$\alpha_{0}=0.6$} & \multicolumn{2}{|c|}{$\alpha_{0}=0.15$} & \multicolumn{2}{|c|}{$\alpha_{0}=0.6$} \\
\hline & slope & $\gamma$ & slope & $\gamma$ & slope & $\gamma$ & slope & $\gamma$ & slope & $\gamma$ & slope & $\gamma$ \\
\hline $0.4-1.4$ & 1.01 & 0.283 & 1.02 & 0.294 & 0.95 & 0.316 & 0.97 & 0.323 & 0.86 & 0.36 & 0.88 & 0.37 \\
\hline $0.2-1.8$ & 1.0 & 0.273 & 1.01 & 0.285 & 0.94 & 0.30 & 0.95 & 0.31 & 0.86 & 0.33 & 0.88 & 0.34 \\
\hline
\end{tabular}

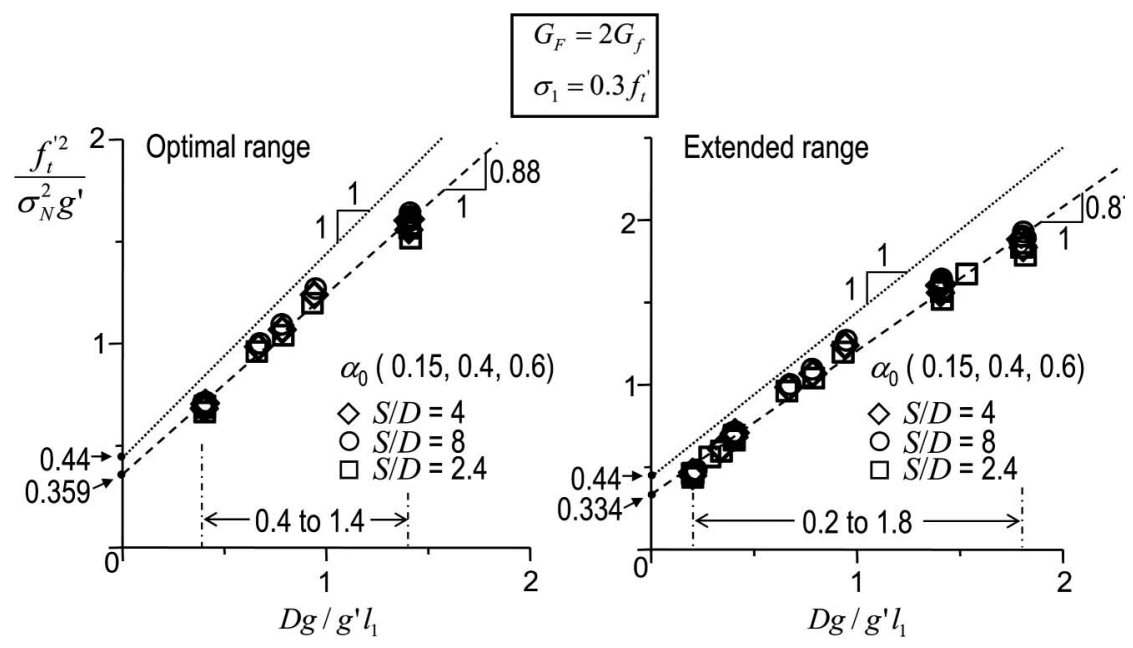

Fig. 7. Slope and intercept obtained by regression for $\sigma_{1}=0.3 f_{t}^{\prime}$ for (a) optimal; and (b) extended size range

is that, for $\sigma_{1} / f_{t}^{\prime}=0.3$, the cohesive stress in the FPZ of smaller specimens enters the tail of the bilinear softening curve, which shortens the transition range.

To investigate the effect of ratio $G_{F} / G_{f}$, the tail slope is varied while keeping $G_{f}, f_{t}^{\prime}$ and $\sigma_{1}$ fixed. It is found that, for a wide range of the tail slopes, $1<G_{F} / G_{f}<5$, which most normal concretes fall into, the aforementioned optimal size range, $0.4<\bar{D}<1.4$, will give good approximations of $G_{f}$ if $\sigma_{1} / f_{t}^{\prime}<0.25$. Fig. 8 shows that increasing $G_{F} / G_{f}$ from 2 to 5 has almost no effect on the regression results for $\sigma_{1} / f_{t}^{\prime}=0.2$. The errors of $G_{f}$ and $f_{t}^{\prime}$ obtained by regression are both less than $1 \%$, which is negligible. However, Fig. 9 shows that a problem arises for the combination of a high knee point and a high ratio $G_{F} / G_{f}$, i.e., $\sigma_{1} / f_{t}^{\prime}=0.25$ and $G_{F} / G_{f}=5$. This combination causes $8 \%$ errors in $G_{f}$ and $7 \%$ in $f_{t}^{\prime}$; see Table 2.

For normal concretes, fortunately, the knee point is usually lower than $0.25 f_{t}^{\prime}$ [Comité Euro-International du Béton-Fédération International du Béton (CEB-FIP) 1991; Wittmann et al. 1988; Rokugo et al. 1989] and the ratio $G_{F} / G_{f}$ is usually less than 5 . Therefore, the optimal size range established here can generally be used in the analysis of size-effect data. Since this optimal size range depends mainly on $G_{f}$ rather than $G_{F}$, it is more appropriate to represent it by the dimensionless shape-independent size $\bar{D}=D g_{0} / g_{0}^{\prime} l_{1}$ than $\hat{D}$ that is based on $G_{F}$. 


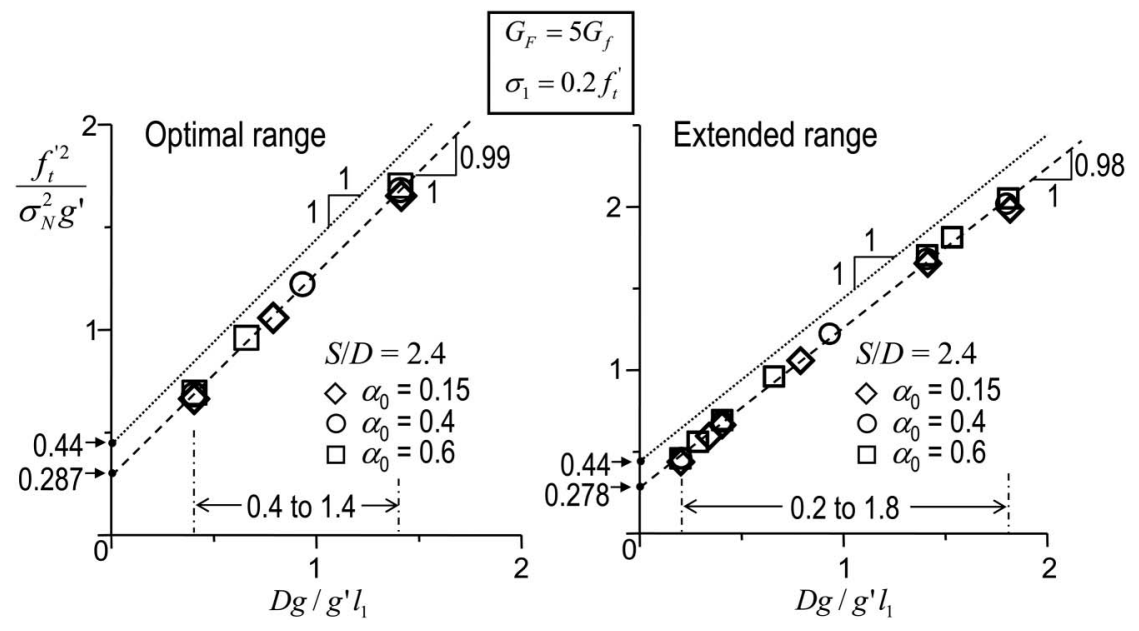

Fig. 8. Slope and intercept obtained by regression for $G_{F}=5 G_{f}$ and $\sigma_{1}=0.2 f_{t}^{\prime}$ for (a) optimal; and (b) extended size range

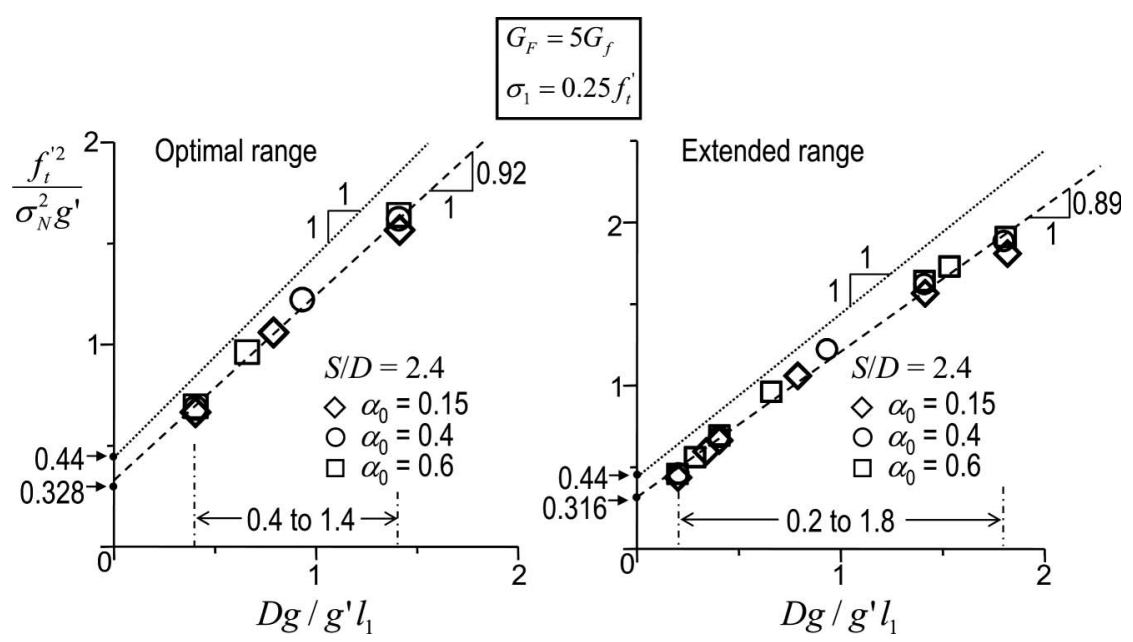

Fig. 9. Slope and intercept obtained by regression for $G_{F}=5 G_{f}$ and $\sigma_{1}=0.25 f_{t}^{\prime}$ for (a) optimal; and (b) extended size range

The minimum breadth of the range of size-effect tests depends on the width of the scatter band (see Fig. 6 in Bažant and Pfeiffer 1987). In the linear plot of Eq. (2), it must exceed this width by a factor of at least 5 to avoid high statistical uncertainty (i.e., excessive COV) of the slope of the regression line (Bažant and Planas 1998). For some materials, such as tough aluminum, the random scatter is so small that the size range of 1:3 is sufficient (Bažant et al. 1987). For the scatter band width typically obtained for concretes, it is desirable to extend the size range to $\bar{D} \in(0.2,1.8)$, which is the size ratio of $1: 9$.

To check whether good results can still be obtained, the program for the cohesive crack model was run for specimens of $\alpha_{0}=0.15$, 0.4 , and 0.6 ; span-depth ratio $S / D=2.4,4$, and $8 ; G_{F} / G_{f}=1,2$, and 5; and beam depths $D$ from $40 \mathrm{~mm}$ to $1,000 \mathrm{~mm}$ (1.57 in. to 39.4 in.). This covers all the notched beam shapes used in laboratory testing. Various linear size-effect regressions were conducted. The parameters obtained are listed in Tables 1 and 2 .

If the size range of specimens is extended to $\bar{D} \in(0.2,1.8)$, which helps to minimize the scatter of the regression slope of test data, both the regression slope and intercept show a small downward shift because of the curvature of the plot outside the optimal interval $\bar{D} \in(0.4,1.4)$. From Tables 1 and 2 , it can be seen the extended size range gives almost same results of $G_{f}$ and $f_{t}^{\prime}$, despite the small downward shift.
Table 2. Effect of $G_{F} / G_{f}$ on Optimal Size Range $\bar{D}, \alpha_{0}$ Ranging from 0.15 to 0.6

\begin{tabular}{|c|c|c|c|c|c|c|c|c|}
\hline \multirow[b]{3}{*}{$\bar{D}$} & \multicolumn{4}{|c|}{$G_{F} / G_{f}=2$} & \multicolumn{4}{|c|}{$G_{F} / G_{f}=5$} \\
\hline & \multicolumn{2}{|c|}{$\sigma_{1}=0.2 f_{t}^{\prime}$} & \multicolumn{2}{|c|}{$\sigma_{1}=0.25 f_{t}^{\prime}$} & \multicolumn{2}{|c|}{$\sigma_{1}=0.2 f_{t}^{\prime}$} & \multicolumn{2}{|c|}{$\sigma_{1}=0.25 f_{t}^{\prime}$} \\
\hline & slope & $\gamma$ & slope & $\gamma$ & slope & $\gamma$ & slope & $\gamma$ \\
\hline $0.4-1.4$ & 1.02 & 0.289 & 0.97 & 0.319 & 0.99 & 0.287 & 0.92 & 0.328 \\
\hline $0.2-1.8$ & 1.01 & 0.28 & 0.95 & 0.31 & 0.98 & 0.278 & 0.89 & 0.316 \\
\hline
\end{tabular}

A proper adjustment of $\gamma$ would permit even broader size ranges, such as 1:16. However, a combination of a high knee point and a long tail, for example, $\sigma_{1}=0.25 f_{t}^{\prime}$ and $G_{F} / G_{f}=5$, will lead to a big deviation (see Table 2). In that case, the broadest acceptable size range is $\bar{D} \in(0.2,1.4)$.

For the extended size range $0.2<\bar{D}<1.8$, linear regression according to the SEL in the classical form [Eq. (1)] will give a good result for the initial fracture energy $G_{f}$, in close agreement with the cohesive crack model. However, to obtain a good result for the tensile strength $f_{t}^{\prime}$ of the cohesive crack model, the correction parameter $\gamma=0.285$, which corresponds to the downward shift of the regression line from the intermediate asymptote, must be taken into account. This leads to the expression $f_{t}^{\prime}=\sqrt{\gamma E G_{f} / c_{f}}$. 
Computational experience shows that the maximum loads of normal size concrete structures depend mainly on $G_{f}$ and $f_{t}^{\prime}$. For that purpose, the size-effect tests appear sufficient. However, to obtain the maximum load of a very large structure or the postpeak response of any structure, which is important to determine the energy absorption capability for dynamic loading, the total fracture energy $G_{F}$ and the knee point $\sigma_{1}$ must also be identified. To this end, the complete postpeak load-deflection curve of notched specimens must be measured, which is discussed next.

\section{Nonuniqueness of Cohesive Softening Law Obtained by Work-of-Fracture Test and Remedy by Size Effect}

In the early stage of concrete fracture research, it has been assumed that the shape of the softening stress-separation curve of the cohesive crack model is unique. An exponential curve (with a downward shift) was proposed and widely considered as adequate. In that case, only two parameters, $G_{F}$ and $f_{t}^{\prime}$, suffice to define the fracture properties, and they would be identifiable either by the work-of-fracture tests on specimens of one size, pioneered for concrete by Hillerborg et al. (1976) and Petersson (1981), or by the size-effect tests. The same would be true for the bilinear softening law if the ratios $G_{F} / G_{f}$ and $\sigma_{1} / f_{t}^{\prime}$ had the same values for all concretes. Unfortunately, these ratios vary from one concrete to another, and so they are unknown material characteristics that need to be measured.

Although the importance of size effect for concrete fracture has been known since 1984, it has usually been tacitly assumed that the bilinear cohesive softening law $\sigma(w)$ can be determined by testing specimens of one size only. However, recent numerical studies at Northwestern University (Yu et al. 2010) show that this is not true. The softening law $\sigma(w)$ of a crack in concrete cannot be uniquely determined from the measured complete load-deflection curve or load-CMOD curve.
The ambiguity of $\sigma(w)$ identified from one-size specimen tests is documented in Fig. 10, where four very different bilinear softening functions $\sigma(w)$ [Fig. 10(c)] are shown to give almost the same load-CMOD curves [Fig. 10(a)]. The four dashed lines in Fig. 10(a), representing four load-CMOD curves of a notched beam of depth $D=215 \mathrm{~mm}$ (8.46 in.), are obtained by using four sets of parameters with tensile strength $f_{t}^{\prime}$ ranging from 4.4 to $7.8 \mathrm{MPa}$ (638 to $1,131 \mathrm{psi}$ ) and the initial fracture energy $G_{f}$ ranging from 21 to $35 \mathrm{~N} / \mathrm{m}(0.12$ to $0.20 \mathrm{lb} /$ in. $)$, whereas the total fracture energy $G_{F}$ is fixed at $64 \mathrm{~N} / \mathrm{m}(0.365 \mathrm{lb} /$ in.) for all the four curves [see Fig. 10(d)]. Although the highest $G_{f}$ value is $68 \%$ larger than the smallest $G_{f}$ value, the differences among the resulting loadCMOD curves are negligible, especially in comparison with the typical random scatter of test results. This further means that the differences in size effect corresponding to different $G_{f}$ values will cause the asymptote of the classical size-effect law to shift horizontally by as much as $\log (0.68)$.

It follows that the work-of-fracture test is insufficient to provide unambiguous basis for the calculation of load capacity of concrete structures, which depends mainly on $G_{f}$ rather than $G_{F}$, unless the structure is extremely large.

Finally, it is interesting to demonstrate in Fig. 10(b) the range of load-CMOD curves when the four aforementioned very different softening laws $\sigma(w)$ are applied to a different specimen size. Consider that $D=25 \mathrm{~mm}$ ( $1 \mathrm{in}$.), which makes the size range close to 1:9. Fig. 10(b) shows that the four softening laws, which gave for $D=215 \mathrm{~mm}$ (8.46 in.) almost the same load-CMOD curves, now give rather different curves. The peak loads differ by as much as $15 \%$.

Therefore, the most efficient way to unambiguously identify the main characteristics of a bilinear cohesive softening law is as follows:

1. Determine the initial fracture energy $G_{f}$, for which the sizeeffect method can be used;

2. Estimate the tensile strength $f_{t}^{\prime}$ by using $\gamma=0.285$; (a)

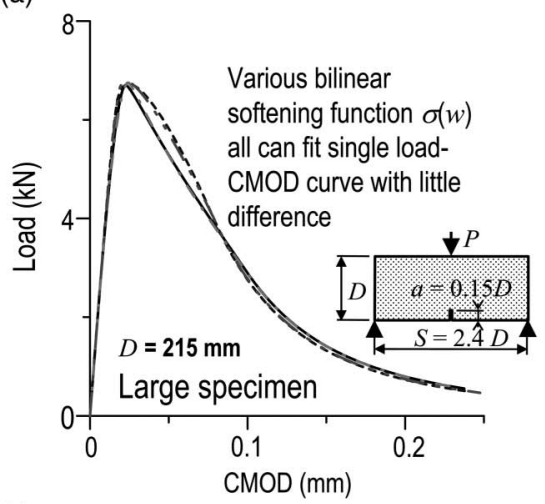

(c)

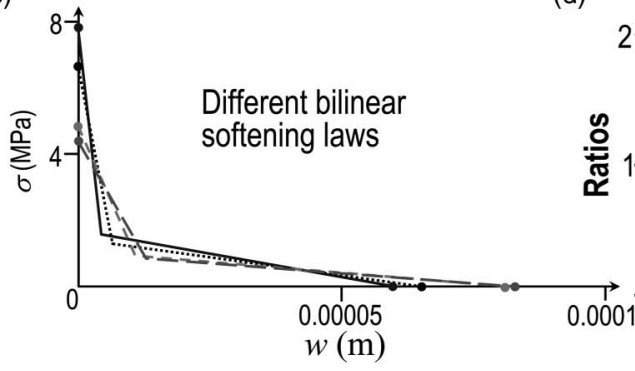

(b)

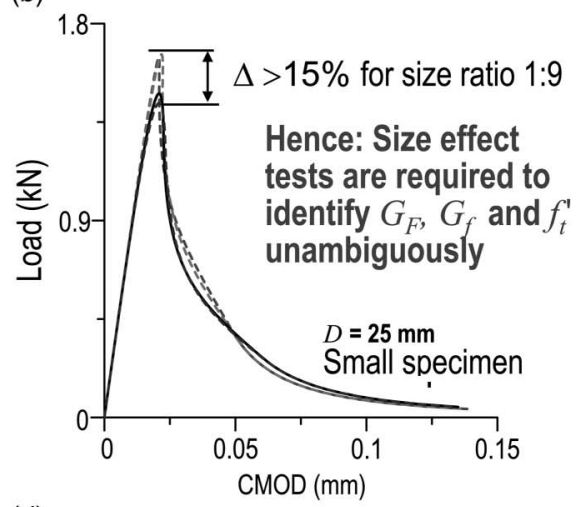

d)

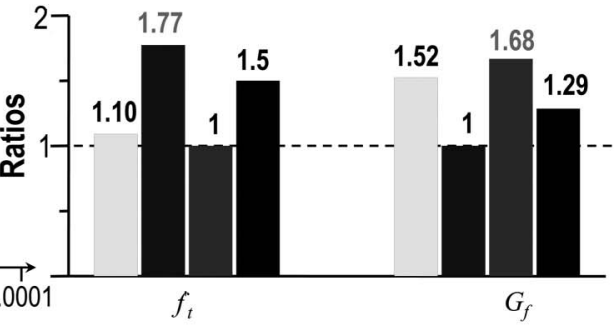

Fig. 10. (a) Load-CMOD curve fitted almost equally by four very different bilinear $\sigma(w)$; (b) substantial deviation happens when size is changed; (c) four different bilinear $\sigma(w)$ used; and (d) the difference in $G_{f}$ and $f_{t}^{\prime}$ 
3. Based on their initial values, select three to five sample points for both $G_{f}$ and $f_{t}^{\prime}$;

4. Run simulations to achieve the optimal fit of the peak loads obtained by size-effect tests (COV of errors $\leq 5 \%$ );

5. Estimate the initial values for total fracture energy $G_{F}$ and knee point $\sigma_{1}$, for example, $G_{F}=2.5 G_{f}$ and $\sigma_{1}=0.2 f_{t}^{\prime}$;

6. Select three to five sample points for both $G_{F}$ and $\sigma_{1}$, then run simulations to obtain the optimal fit of the measured loadCMOD curves (COV of errors $\leq 5 \%$ ); and

7. Repeat the preceding steps until an acceptable error $(\mathrm{CoV} \leq 5 \%)$ is achieved for both the peak load and the load-CMOD curve.

It thus becomes obvious that size-effect testing is indispensable for identifying the fracture properties of concrete uniquely. Only the cohesive softening law $\sigma(w)$ that yields a close match of both the load-deflection (or load-CMOD) curve and the size-effect curve of a sufficient size range can be regarded as a realistic representation of the fracture properties.

The absence of size-effect testing may explain why the $G_{F}$ values in the database compiled from the literature (Bažant and Becq-Giraudon 2002) exhibit such a huge random variability, with the coefficient of variation of $G_{F}$ being more than twice as large as that of $G_{f}$.

\section{Simplification Based on Estimating Ratios $G_{F}=G_{f}$ and $\sigma_{1}=f_{t}^{\prime}$}

Enhancing the size-effect tests by work-of-fracture tests and vice versa would not be necessary if the aforementioned ratios had fixed values or could be reasonably estimated from concrete composition. On the basis of some limited experience, the following ratios have been proposed:

$$
G_{F} / G_{f} \approx 2.5, \quad \sigma_{1} / f_{t}^{\prime} \approx 0.25
$$

Although the use of these estimates leads to a better fracture analysis of structures than does the use of linear or exponential softening, these estimates are, unfortunately, very crude; the $G_{F} / G_{f}$ ratio probably varies from less than 2 to approximately 5 , and the ratio $\sigma_{1} / f_{t}^{\prime}$ from 0.15 to 0.3 .

Because the COV of the scatter of $G_{F}$ measured by the work-offracture method is more than twice as large as that of $G_{f}$ measured by the size-effect method (Bažant and Becq-Giraudon 2002), it is better to predict $G_{F}$ from $G_{f}$ than vice versa. Thus, if only one type of fracture test must be conducted, it is better to choose the size-effect tests than the work-of-fracture tests.

\section{Conclusions}

1. The optimal size range for identifying the initial fracture energy and tensile strength of cohesive crack model is $\bar{D} \in(0.2,1.8)$. For normal concretes, this size range is applicable as long as the knee point of the bilinear softening curve is not higher than $25 \%$ of the tensile strength, and the ratio of total to initial fracture energy is not greater than 5 .

2. If not supplemented by size-effect tests, the values of initial fracture energy identified from the tests of load-deflection curves of notched specimens of one size only can differ by as much as $68 \%$.

3. To identify the cohesive fracture characteristics of concrete and other quasibrittle materials, tests of the size effect are indispensable. The work-of-fracture test of specimens of one size is insufficient.

\section{Acknowledgments}

Support by the U.S. Department of Transportation through Grant 20778 from the Infrastructure Technology Institute of Northwestern University is gratefully acknowledged.

\section{Reference}

Barenblatt, G. I. (1959). "The formulation of equilibrium cracks during brittle fracture, general ideas and hypothesis, axially symmetric cracks." Prikl. Mat. Mech., 23(3), 434-444.

Barenblatt, G. I. (1962). "The mathematical theory of equilibrium of cracks in brittle failure." Adv. Appl. Mech., 7, 55-129.

Barenblatt, G. I. (1996). Scaling, selfsimilarity and intermediate asymptotics, Cambridge University Press, Cambridge, UK.

Barenblatt, G. I. (2003). Scaling, Cambridge University Press, Cambridge, UK.

Bažant, Z. P. (1976). "Instability, ductility, and size effect in strainsoftening concrete." J. Eng. Mech. Div., 102(2), 331-344.

Bažant, Z. P. (1977). "Discussion of 'Instability, ductility, and size effect in strain-softening concrete' by Guillermo J. Creus." J. Eng. Mech. Div., 103(2), 357-358.

Bažant, Z. P. (1977). "Discussion of 'Instability, ductility, and size effect in strain-softening concrete' by Lorenzo Jurina." J. Eng. Mech. Div., 103(4), 775-777.

Bažant, Z. P. (1978). "Closure of 'Instability, ductility, and size effect in strain-softening concrete'.” J. Eng. Mech. Div., 104(2), 501-502.

Bažant, Z. P. (1984). "Size effect in blunt fracture: Concrete, rock, metal." J. Eng. Mech., 110(4), 518-535.

Bažant, Z. P. (1997). "Scaling of quasibrittle fracture: Asymptotic analysis." Int. J. Fract., 83(1), 19-40.

Bažant, Z. P. (2004). "Scaling theory for quaisbrittle structural failure." Proc. Natl. Acad. Sci. U.S.A., 101(37), 13400.

Bažant, Z. P. (2005). Scaling of structural strength, 2nd Ed., Elsevier, London.

Bažant, Z. P., and Becq-Giraudon, E. (2002). "Statistical prediction of fracture parameters of concrete and implications for choice of testing standard." Cem. Concr. Res., 32(4), 529-556.

Bažant, Z. P., and Kazemi, M. T. (1990a). "Determination of fracture energy, process zone length and brittleness number from size effect, with application to rock and concrete." Int. J. Fract., 44, 111-131.

Bažant, Z. P., and Kazemi, M. T. (1990b). "Size effect in fracture of ceramics and its use to determine fracture energy and effective process zone length." J. Am. Ceram. Soc., 73(7), 1841-1853.

Bažant, Z. P., and Kazemi, M. T. (1991). "Size effect on diagonal shear failure of beams without stirrups." ACI Struct. J., 88(3), 268-276.

Bažant, Z. P., Lee, S.-G., and Pfeiffer, P. A. (1987). "Size effect tests and fracture characteristics of aluminum." Eng. Fract. Mech., 26(1), 45-57.

Bažant, Z. P., and Oh, B.-H. (1983). "Crack band theory for fracture of concrete." Mater. Struct., 16(3), 155-177.

Bažant, Z. P., and Pfeiffer, P. A. (1987). "Determination of fracture energy from size effect and brittleness number." ACI Mater. J., 84(6), 463-480.

Bažant, Z. P., and Planas, J. (1998). Fracture and size effect in concrete and other quasibrittle materials, CRC Press.

Bažant, Z. P., Yu, Q., and Zi, G. (2002). "Choice of standard fracture test for concrete and its statistical evaluation." Int. J. Fract., 118, 303-337.

Comité Euro-International du Béton-Fédération International du Béton (CEB-FIP). (1991). "Bulletin d'information du Comité EuroInternational du Béton." CEB-FIP model code 1990, final draft, 203-205, Laussane, Switzerland.

Cusatis, G., and Schauffert, E. A. (2009). "Cohesive crack analysis of size effect." Eng. Fract. Mech., 76(14), 2163-2173.

Hillerborg, A., Modéer, M., and Petersson, P. E. (1976). "Analysis of crack formation and crack growth in concrete by means of fracture mechanics and finite elements." Cem. Concr. Res., 6(6), 773-782.

Irwin, G. R. (1958). "Fracture." In Handbuch der Physik, Flügge, ed., Springer-Verlag, Berlin, Vol. 6, 551-590. 
Nakayama, J. (1965). "Direct measurement of fracture energies of brittle heterogeneous material." J. Am. Ceram. Soc., 48(11), 583-587.

Petersson, P. E. (1981). "Crack growth and development of fracture zone in plain concrete and similar materials." Rep. No. TVBM-1006, Division of Building Materials, Lund Institute of Technology, Lund, Sweden.

Rice, J. R. (1968). Mathematical analysis in the mechanics of fractureAn advanced treatise, Vol. 2, H. Liebowitz, ed., Academic Press, New York, 191-308.

RILEM. (1985). "Determination of the fracture energy of mortar and concrete by means of three-point bend tests on notched beams." Mater. Struct., 18(4), 285-290.

RILEM. (1990). "Size-effect method for determining fracture energy and process zone size of concrete." Mater. Struct., 23, 461-465.

RILEM TC QFS. (2004). "Quasibrittle fracture scaling and size effectFinal rep." Mater. Struct., 37(272), 547-586.

Rokugo, K., Iwasa, M., Suzuki, T., and Koyanagi, W. (1989). "Testing methods to determine tensile strain softening curve and fracture energy of concrete." Fracture toughness and fracture energy: Test methods for concrete and rock, $\mathrm{H}$. Mihashi, $\mathrm{H}$. Takahashi, and F. H. Wittmann, eds., Balkema, Rotterdam, 153-163.

Tatersall, H. G., and Tapin, G. (1966). "The work of fracture and its measurement in metals, ceramics and other materials." J. Mater. Sci., 1(3), 296-301.

Walsh, P. E. (1972). "Fracture of plain concrete." Indian Concr. J., 46(11), 469-476.

Walsh, P. E. (1976). "Crack initiation in plain concrete." Mag. Concr. Res., 28(94), 37-41.

Weibull, W. (1939). "The phenomenon of rupture in solids." Proc., Royal Swedish Institute of Engineering Research, 153, Stockholm, 1-55.

Weibull, W. (1951). "A statistical distribution function of wide applicability." J. Appl. Mech., 18, 293-297.

Wittmann, F. H., Rokugo, K., Brühwiler, E., Mihashi, H., and Simonin, P. (1988). "Fracture energy and strain softening of concrete as determined by means of compact tension specimens." Mater. Struct., 21(1), 21-32.

Yu, Q., Le, J.-L., Hoover, C. G., and Bažant, Z. P. (2010). "Problems with Hu-Duan boundary effect model and its comparison to size-shape effect law for quasi-brittle fracture." J. Eng. Mech., 136(1), 40-50. 